\title{
Building a Learning Collaborative
}

Susan Adams, RN, PhD, Marita G. Titler, RN, PhD, FAAN

\begin{abstract}
Background: Development and dissemination of evidence-based practice (EBP) guidelines are important first steps, but without active translation efforts, they do little to promote knowledge uptake by direct care providers. In order to close the gap between discovery and use of knowledge, efforts should focus on methods to speed translation of research findings into practice.

Aim: To facilitate this process, the National Nursing Practice Network (NNPN) was established to provide participating health care organizations with access to resources, information, opportunities for learning and knowledge transfer, and legitimacy and credibility with internal and external stakeholders.

Approach: Designed as a practice collaborative learning network, the NNPN uses an interactive learning approach by valuing and learning from the experiences of members and encouraging active participation through conferences, teleconferencing, and use of an interactive member Web site. Use of a network of acute care sites to increase the uptake of EBP is based on social network theory; the NNPN is a network of acute care hospitals through which data, information, knowledge, practices, and behaviors flow.

Discussion and Conclusion: The use of a collaborative nursing network provides an innovative way to address the difficulty of implementing and sustaining EBP in organizations and also for maximizing resources and knowledge. Early evaluation data are promising. This approach is offered as one possible method of moving use of EBP in nursing forward.

KEYWORDS learning collaborative, evidence-based practice (EBP), nursing, community of practice
\end{abstract}

Worldviews on Evidence-Based Nursing 2010; 7(3):165-173. Copyright @2010 Sigma Theta Tau International

\section{BUILDING A LEARNING COLLABORATIVE}

$\mathrm{T}$ here is a growing awareness by nurses throughout the international community about the importance of basing nursing practice on the best available evidence, and numerous resources are available to provide access to research evidence. However, in spite of improved access to the growing evidence base, there remains inconsistent use of evidence-based health care practices at the point of care. One of the greatest challenges facing nurses is how to ef-

Susan Adams, Director, National Nursing Practice Network, Associate Director, Research Translation and Dissemination Core, University of Iowa College of Nursing, Iowa City, IA. Marita G. Titler, Executive Director, National Nursing Practice Network, Professor, Associate Dean for Practice and Clinical Scholarship Development, and the Rhetaugh Dumas Endowed Chair, University of Michigan School of Nursing and UMHS, Ann Arbor, MI

Address correspondence to Susan Adams, University of Iowa College of Nursing 4116 Westlawn, Iowa City, IA 52242; susan-adams@uiowa.edu

The authors acknowledge and thank the NNPN steering committee for their contributions in developing the NNPN: Roxanne Wilson, RN, MS; Cristy Bledsoe, RN, BSN, MSN, PhD; Kay Takes, RN, BSN, MA, CNAA; Nancy Weber, RN, MSN; Susan Lacey, RN, PhD; and Tina Lund, RN, MSN, CNAA.

Accepted 3 August 2009

Copyright $@ 2009$ Sigma Theta Tau International

1545-102X1/09 fectively and efficiently implement and sustain evidencebased health care practices in their practice settings (Cullen $\&$ Titler 2004; Cullen et al. 2005; Stetler et al. 2006).

Use of evidence in practice is facilitated, in part, by modeling and imitation of others who have successfully adopted such practices, an organizational culture that values and supports use of evidence, and localization of the evidence for use in a specific health care setting (Titler \& Everett 2001). Yet, much work is needed to find practical and effective methods to implement these strategies on a broad scale.

\section{BACKGROUND}

Within the United States, the Research Translation and Dissemination Core (RTDC) of the federally funded Gerontological Nursing Interventions Research Center, under the direction of Marita Titler, has a long history of providing education and mentoring of nurses throughout the international community. Formed in 1994, the RTDC began the development and distribution of evidence-based practice (EBP) guidelines for nurses on topics applicable to care of older adults, administrative guidelines, and most 
recently, guidelines for school health. Over 13,000 of these guidelines have been disseminated nationally and internationally, and over 50,000 permissions to copy have been granted. In addition, permission has been given for translation of guidelines into Chinese, Japanese, Spanish, and Swedish.

Because the availability of EBP guidelines does not ensure use in clinical practice, in 2002 the University of Iowa Hospitals and Clinics (UIHC), in partnership with the RTDC and the University of Iowa's College of Nursing, began looking for new methods to assist health care providers with translating evidence-based guidelines into practice. The Toolkit for Promoting EBP (Titler 2002) was developed to guide health care leaders in implementing evidence-based health care in their setting. This toolkit provides information on (1) selecting a topic for implementation, (2) finding and critiquing evidence, (3) formulating an action plan, and (4) trying and evaluating the change in practice. At the same time, the Staff Nurse Internship Program was initiated at UIHC for staff nurses to learn EBP by completing an EBP project with mentorship from experts within the organization.

The same year, the Advanced Practice Institute (API): Promoting Adoption of EBP was initiated as a collaborative effort between the University of Iowa College of Nursing and UIHC. This intensive 3-day institute offered on-site at UIHC, educates nursing leaders from outside the UIHC system about effective ways to guide colleagues and staff in the integration of evidence-based knowledge into practice. The API uses a combination of didactic teaching, interactive work sessions, consultation with experts, and networking to increase the learning experience. The popularity of this institute resulted in requests for an increased number of training sessions per year offered on-site at the University of Iowa and a growing number of requests for API staff to travel for intensive training programs at hospitals both nationally and internationally. Because of the increasing interest of nursing leaders and administrators in improving patient outcomes through learning activities provided by the UIHC and RTDC, new strategies were needed to maximize learning opportunities and resources.

\section{LEARNING COLLABORATIVES}

Using a learning collaborative to increase the implementation of quality improvement (QI) initiatives has become increasingly popular, the most well-known example being the Institute for Health care Improvement (IHI), an international, independent not-for-profit organization that is focused on helping health care systems improve patient outcomes (Berwick 2003; Wilson et al. 2003; Mittman 2004). While the term learning collaborative is relatively new, the idea is not. Learning collaboratives build on the concept of communities of practice (COP). Wenger describes $\mathrm{COP}$ as people coming together to engage in a process of collective learning in a shared domain of interest (Wenger et al. 2002). A COP is distinguished from communities in general by meeting three criteria: (1) a shared domain of interest, (2) engagement in joint activities and discussions, assisting each other and sharing information, and (3) a common practice as evidenced by sharing a similar set of resources (e.g., experience, ways of addressing recurring problems, tools, and expertise and knowledge of the shared domain) (Wenger 1998).

COPs come in many forms and are known by different names, such as learning networks or learning cooperatives (Wenger 1998; Lesser \& Storck 2001; Wenger et al. 2002). True COPs differ from the QI team format used in U.S. hospitals and promoted by IHI in several important ways. COP relationships form voluntarily around practice; in contrast, team relationships for QI learning collaboratives are assigned by the organization. In the same way, QI collaboratives have hierarchical authority structures that are predetermined by the organization, yet the authority and legitimacy in COPs emerge through expertise around areas of practice. The goals of QI teams are most likely not determined by the team members, but rather by the organization. In contrast, COPs are responsible to their own members, set their own goals, and develop their own work and reporting processes through an egalitarian approach by providing equal access to resources and to decisionmaking power (Lesser \& Storck 2001). While additional rigorous empirical testing is still needed to determine how variations in learning collaboratives affect improvement in patient outcomes (Wilson et al. 2003; Mittman 2004), evidence of their effectiveness in improving patient outcomes and maximizing resources is emerging (Lesser \& Storck 2001; Ayers et al. 2005; Fremont et al. 2006; Young et al. 2006).

\section{INITIATING THE NATIONAL NURSING PRACTICE NETWORK}

In 2005, in an effort to advance the use of evidence-based nursing to a broader audience and make the most efficient use of available resources, the National Nursing Practice Network (NNPN) was initiated based on a community of practice collaborative learning model designed to translate evidence into practice. Several reasons prompted the decision to design the NNPN as a network specifically for nurses employed in hospitals. First, although the majority of nurses in the United States work in acute care settings and are the largest segment of health care workers in that setting, existing learning collaboratives in acute 
care have not focused on nurses as a group, but rather on interdisciplinary teams implementing specific QI projects. While these interdisciplinary teams are necessary and valued, the teams involved in these projects are disbanded after the project is complete. As a result, nurses do not receive ongoing mentoring, support, role modeling, and professional development regarding EBP and implementation after the conclusion of the project. Connecting nurses across acute care settings in a continuous, stable collaborative provides a method of impacting nursing practice on a large scale by developing ongoing relationships and sustaining enthusiasm and momentum that may be lost when interdisciplinary teams are disbanded at the end of a project.

Second, the practice-based networks funded by the Agency for Healthcare Research and Quality (AHRQ), the U.S. federal entity charged with improving quality, safety, efficiency, and effectiveness of health care for Americans, are made up of primary care clinics and focus on physician practices. A network of nurses that focuses on nursing practice in hospitals is needed to share information of EBPs and implementation strategies so that nurses can leverage lessons learned to make excellence in patient care a reality (Needleman et al. 2006). Use of the NNPN to bring together nurses from diverse types of hospitals (local community hospitals, academic medical centers, Veteran Affairs medical centers, small, large, rural, and urban) affords members an opportunity to learn what has worked well in terms of implementing evidence-based nursing practices in other hospitals and makes implementation of EBPs more efficient. Last, the NNPN addresses organizational infrastructures within hospitals that directly affect nurses (e.g., integration of EBPs in nursing governance structures) to support the work of implementing EBPs. Nursing administrators across hospitals can discuss and compare effective models of nursing governance, models of care, models of EBP implementation, and other topics specific to nursing needs.

In February 2005, nurse leaders from 25 selected hospitals across the United States were invited to come together to develop a network dedicated to improving patient outcomes through use of EBP. Original sites were selected based on existing relationships developed through (1) participation in the original AHRQ Translating Research into Practice Study in which Titler was the primary investigator or (2) participation in multiple Advanced Practice Institutes at UIHC. The response was overwhelmingly positive, with 20 additional hospitals requesting permission to join after hearing about the new venture. Along with the letter of invitation, these 45 hospitals received two questionnaires: a request for demographic data, and a request to prioritize topics of interest for their organization.
Admission into the NNPN requires the approval of the Chief Nursing Officer (CNO) of the hospital. Each hospital in the NNPN is considered a member and all nurses in the member hospital have full access to the services provided by the NNPN. The CNO, or designee, serves as the primary representative. Each hospital receives one vote or one contributing document (e.g., needs survey), completed by the $\mathrm{CNO}$ or designee. Original sites were geographically located in 15 states in the United States. These sites represented small ( $<100$ acute care beds, $N=9$ ), medium (101400 acute care beds, $N=27$ ) and large ( $>400$ acute care beds, $N=9$ ) hospitals, and included 4 academic medical centers, 8 Veterans Affairs (VA) Medical Centers, and 33 local community hospitals that are public or private nonprofit. Most of these acute care hospitals were affiliated with skilled, long-term care facilities, ambulatory services, and primary care sites.

\section{Priority Needs Survey}

The CNO or designee of each new member organization was asked to rate on a Likert scale $(1=$ low to $5=$ high $)$ the priority of major topics of interest according to the needs of the organization. Rated as high priority were: evaluating outcomes to show impact of EBP (85.3\%); training in EBPs with practical examples (88.2\%); and sustaining momentum for EBPs, especially among staff nurses $(82.3 \%)$. Areas of specific educational training requested included: basic EBP courses for staff nurses (76.4\%), advanced level courses $(70.5 \%)$, and training for senior leadership (58.8\%). When asked to select the top five clinical topic areas of highest interest for the organization, measuring patient outcomes was the highest need, followed by building a culture of safety, acute pain management, culturally competent care, and failure to rescue (Titler $\&$ Adams 2007, unpublished data).

\section{Creation of the Steering Committee and Workgroups}

A request was sent out in late 2005 for interested applicants to serve on a steering committee. A panel of experts selected the committee, comprising multiregional NNPN members, from a pool of highly motivated and qualified applicants to facilitate decision making and the development of a strategic plan. Steering committee members were selected to provide a wide range of expertise, experience, and representativeness of the variety of organizations in the NNPN. The nine-member steering committee began meeting in January 2006 with monthly conference calls and additional electronic communications. This committee developed a mission, vision, and strategic plan based on the needs assessment, which was then ratified by the NNPN community as a whole. Based on the needs assessment, the steering committee proposed the development 
Building a Learning Collaborative

TABLE 1

NNPN workgroup mission statements

\begin{tabular}{lr}
\hline WORKGROUP & MISSION STATEMENT \\
\hline $\begin{array}{l}\text { Education } \\
\text { Networking }\end{array}$ & $\begin{array}{r}\text { To provide a variety of educational programming on evidenced-based practice to members of the NNPN. } \\
\text { To provide environments that encourage and support communication in the pursuit of evidence-based health } \\
\text { care practices. }\end{array}$ \\
$\begin{array}{l}\text { Tommunity-based } \\
\text { participatory research }\end{array}$ & $\begin{array}{l}\text { based practices (EBPs), and to provide a consortium of sites for research in clinical care and translation } \\
\text { science. }\end{array}$ \\
Implementation & $\begin{array}{r}\text { To provide tools, evidence-based processes, and mentoring to maximize implementation efforts by NNPN } \\
\text { members, both individually and organizationally. }\end{array}$ \\
\hline
\end{tabular}

of workgroups in four conceptual areas, each chaired by a steering committee member and made up of NNPN member volunteers. These four conceptual areas were designed to foster sharing resources and knowledge across hospitals and to provide an opportunity for hospitals to participate in research about nursing practice (See Table 1).

\section{First NNPN Invitational Conference}

The initial work of the NNPN steering committee, NNPN nurse representatives and workgroup members culminated on 18-19 January 2007 in the first NNPN invitational conference. Attendees to this conference included steering committee members, CNOs, members of each workgroup, and NNPN nurse representatives from all NNPN organizations. This conference, funded by the AHRQ, met at the Nursing Clinical Education Center within the UIHC in Iowa City. Attendees discussed the strategic plan, identified short and long-term goals of the network, and worked to develop an action plan to focus resources on meeting the top priorities of the NNPN. Conference presentations, presented by AHRQ representatives, focused on developing a participatory network, with much of the time spent in break-out discussions and workgroups. Attending representatives of the NNPN organizations emphasized the need for making quality indicators a top priority in addition to those identified in the priority survey, citing the difficulty in understanding and consolidating the strength of evidence behind the indicators and needing effective methods to achieve meeting these benchmarks. The NNPN attendees also requested help in developing and accessing evidence-based quality indicator measures that are nurse sensitive.

\section{Refinement of the Strategic Plan}

Organizations had identified several areas of need in the initial priority needs survey and refined those needs during the conference. A top priority was a request for help in developing training programs for nurses in EBP for all levels (from basic to advanced), along with practical examples.
Members also requested educational training that would be appropriate for both staff nurses working at the point of care, as well as nurse leaders, and hospital administrators. There was great interest in methods to sustain the momentum for EBP, especially with staff nurses, and methods to evaluate outcomes to show the impact on care. Other areas of high interest included a convenient "one-stop" Web site to provide needed information and networking opportunities, and the development of toolkits for implementing EBP at both the individual and organizational level. Participants at the NNPN conference used brainstorming techniques in small groups to answer various questions provided to stimulate ideas. Summations are provided in Tables 2 and 3.

Outcomes of the conference included a refinement of the strategic plan based on the results of the individual group work and subsequent joint discussions, and the development of a dynamic action plan for each of the four workgroups. An overwhelming number of attendees requested annual or semiannual face-to-face meetings to continue the momentum and update priorities.

\section{PROGRESS TO DATE}

February 2009 marked the fourth anniversary of the NNPN. Word-of-mouth communication resulted in a growing waiting list of requests to join. In 2008, as added infrastructure support became available from the VA Center for Research in the Implementation of Innovative Strategies in Practice (CRIISP), 20 additional sites were added. At the current time, the NNPN is made up of 70 hospitals in 26 U.S. states and Puerto Rico, and additional hospitals in Singapore and Pakistan. Since the first invitational NNPN conference, NNPN member organizations, workgroups, and steering committee have worked diligently to accomplish the goals identified during the conference. The Networking Workgroup has developed the NNPN Web site (www.nnpnetwork.org) and is continually working to expand and improve its usefulness. 
TABLE 2

Priorities for acute care organizations

\section{WHAT ARE THE TOP PRIORITIES FOR ACUTE CARE ORGANIZATIONS? WHAT ARE YOUR MOST IMMEDIATE NEEDS?}

1. To build a foundation of understanding of what is required for translation/implementation.

2. Training guides for implementation.

3. Prioritization and strength of evidence behind quality measures (e.g., U.S. National Database of Nursing Quality Indicators, AHRO, U.S. National Quality Forum).

4. EBP accessible/useable at the point of care.

5. How to form an infrastructure to support EBP.

6. Patient safety, including medication reconciliation, falls, standardized care protocols, nurse satisfaction, fiscal constraints, performance measures for employees and leaders (competencies).

7. Standardized protocols for care.

8. How to make a business case for EBP.

9. Central repository for nursing EBP.

10. Increased involvement and empowerment of the bedside nurse.

\section{TABLE 3}

Benefits of participation in NNPN

\section{WHAT BENEFITS WOULD YOU AND YOUR ORGANIZATION LIKE TO RECEIVE BY YOUR PARTICIPATION IN NNPN?}

1. Quick and ready access to reliable evidence the bedside RN can easily use.

2. Methods to communicate with other members-networking and sharing best practice ideas and methods to overcome barriers.

3. Toolkits: Resources that would be available to all members. Members should be able to customize them for their institution.

4. Information and help on implementation-what has worked and what has not.

5. Help on evaluation of implementation. What metrics/measurement should we use to gauge how well a new innovation was measured?

6. Members can post/share strategies they have used to meet regulatory or quality standards to reduce time, efforts and redundancy.

7. Opportunity for nurses at the bedside to collaborate and be part of a powerful force for change - to drive the change.

8. Generate collaboration models for education.

9. Ability to produce a larger body of EBP.

10. Improved rapid cycling of EBP information.

11. Standardization of nursing practice.

12. Nursing to own its practice and related issues (i.e., nurses would be in charge of improving their practice and their own professional growth, and not dependent on the "permission" of other stakeholders to improve nursing care).

13. Commitment to nursing outside of our own organization (i.e., nurses will help the nursing community and improving patient outcomes as a whole, and not be solely concerned with local issues).

14. Provide a forum for members to solve problems.

15. NNPN should be the gold standard for EBP (i.e., the NNPN will set the highest standard for nursing practice regarding EBP).

16. Showcase the intellectual capital of nurses (i.e., the NNPN will provide a venue to showcase the work that nurses have created and completed and this will not be hidden within a unit, but shared across the NNPN for all to see) .

17. Practice in providing mentoring support to nurses.

In spring 2008, staff nurses and nurse managers at four NNPN hospital sites participated in an assessment of the ease of use and overall usefulness of the content of the Web site. Sites were selected based on location, specifically the proximity to the University of Iowa, and variation in the number of beds. Four sizes of facilities were chosen to reach nurses who work in a variety of service settings. It was important to meet with at least one individual from each position at each site to provide information from both working nurses and nurse managers.
Interviews allowed nurses $(n=6)$ and nurse managers $(n=6)$ an opportunity to use and comment on the NNPN Web site. The interviews followed a three-step process. First, participating nurses were asked about their information needs and expectations of the Web site. This provided insight into the type of information nurses would expect to see on a Web site. Second, nurses were given access to the Web site and were asked to perform a self-selected search and two additional, standardized tasks. Observations and documentation of how the nurses used the Web 
site was tracked. Third, nurses were asked about their reaction to the site, their intended usage, and their suggestions for improvement. This feedback resulted in renovations and additions to the site, including the current development of a new section exclusively focused on the needs of the staff nurses. It is designed to provide quick, up-to-date information on topics of interest for practicing nurses.

The Web site is designed to be constantly changing. Nurses throughout the NNPN contribute the content, and make suggestions for new additions. Members are encouraged to share expertise, successes and failures, along with thoughts, ideas, and lessons learned with other nurses throughout the network. Included are an online discussion board, information, educational modules, and multiple projects implemented in NNPN sites. The Web site has developed a search feature that allows nurses to quickly search for EBP articles, information, or EBP guidelines on topics of interest. Because the search engine looks only for information on preapproved EBP sites, the process is streamlined for busy nurses seeking reliable evidence at the point of care.

The Education Workgroup, along with input from member sites, has completed 11 educational modules that are available on the NNPN Web site, with more under development. Modules include introductory and advanced EBP content. Some modules contain audio and others are designed to be easily adapted for presentation in member organizations with notes for presenters. Also included is a community-based module for introducing school nurses to EBP, promoting collaboration across the larger nursing community. Five educational modules under development at this time are designed to meet member requests: EBP for senior leadership; implementation strategies; QI data analysis and interpretation; elementary statistics; and making the business case for using EBP. A minimum of two new modules will be added annually.

In 2009, the Education Workgroup established a Journal Club through the NNPN Web site. The online journal club features articles on how to start a journal club, forms to use when critiquing articles, completed online article critiques, and an opportunity to share journal club experiences with nurses in our member sites. Member sites may suggest articles for critique. Experienced journal club mentors are available for assistance via e-mail or phone communication. The NNPN also features a live interactive journal club discussion group offered bimonthly. Interested nurses from the member hospitals are invited to read and critique a selected article, and come together to discuss the article in a Web-based interactive session.

The Community-Based Participatory Research (CBPR) Workgroup seeks opportunities to facilitate research that addresses questions of interest to the hospitals in the NNPN community and uses a CBPR model for any research conducted in the NNPN community. The CBPR community is defined as nurses working in NNPN hospitals who share common questions about improving patient outcomes. The CBPR Workgroup is committed to understanding the clinical questions of acute care practice and how these questions might be addressed through application of evidence and/or conduct of research.

NNPN sites are encouraged to become involved in the CBPR by informing workgroup members of needs in their organization, and may volunteer to participate in ongoing studies of interest. The CBPR workgroup members and NNPN staff seek funding opportunities that specifically meet these requested needs of the members, and provide the needed expertise for grant writing and the conduct of research. As sites voluntarily participate in research, the NNPN is collectively able to address critical clinical issues that nurses face every day.

In 2007, an opportunity to participate in the Robert Wood Johnson Foundation (RWJ) funded Interdisciplinary Nursing Quality Research Initiative (INQRI) study was presented to our members and the majority of the NNPN sites joined the study, resulting in participation of 190 nursing care units in 45 U.S. hospitals. The study will examine a number of factors, which may influence the effectiveness of nursing interventions regarding the prevalence of patient falls and injuries from falls. For instance, the team will look at skill mix, turnover, and nurse empowerment within an organization in relation to hospital size, and $\mathrm{RN}$ vacancy rate. Researchers will also examine the contribution of fall prevention processes (e.g., risk assessment), and hospital structure efforts (e.g., interdisciplinary fall prevention teams) on fall prevalence and injuries from falls at two points in time over 18 months. The study further aims to determine whether the level of professional nursing practice has an impact on adoption of EBPs for fall prevention. These sites reflect wide geographic distribution and hospital size, thereby increasing understanding of the challenges, benefits, and feasibility of using nurse-sensitive performance measures. Funding for this study was pursued as a direct result of requests during the Invitational Conference to provide help in identifying nurse-sensitive indicators that impact National Database of Nursing Quality Indicators measures.

Member sites will also be participating in the American Organization of Nursing Executives (AONE) funded study Examining Nurse Manager Behaviors Related to Use of Evidence-Based Practices on Nursing Units. This qualitative study is designed to explore nurse managers' administrative and leadership behaviors regarding the integration and promotion of EBPs, and the resulting impact on patient 
care. The researchers will employ semistructured interviews to better understand these behaviors, and will ultimately analyze the perceived gap between high-performing and low-performing nursing units.

In addition, a tool to measure context variables that impact EBP use is being developed at the request of our members to assess interested NNPN sites. This tool will be used to provide feedback to organizations and to tailor intensive interventions to improve EBP uptake. Four member sites have volunteered to participate in psychometric testing of the tool.

The Implementation Workgroup has developed an Organizational Implementation Toolkit for our members to provide guidance in developing a culture of EBP at the organizational level. To supplement the Toolkit, summaries of an array of instruments for measuring core health care delivery context variables are posted on the Web site. These core variables that make up the organizational context include culture and climate, interactive human relationships, and the measurement and evaluation processes specific to organizations. While there is no general consensus on which set of core elements are key to promoting EBP, various instruments will be reviewed that measure those core elements suggested in the literature that influence the rate and sustainability of EBP. Instrument description, psychometric data, references, and sources are provided.

Member organizations have contributed to the NNPN by sending EBP projects, posters, abstracts, PowerPoint presentations, and news from their organizations for posting on the Web site, and by providing volunteers for workgroups and the steering committee. Sample EBP project reports provide real success stories from a number of member organizations. Each project report provides an opportunity to see the process and outcomes that provide opportunities for discussion and shared learning among members. Member organizations have also contributed educational modules used in their organizations on such topics as introduction to EBP, evidence-based tracheostomy care, and the implementation of a pneumovax vaccination program.

In the spring of 2008, the NNPN began a series of small group-phone conferences to increase networking in the growing organization, and to understand where individual organizations were in their EBP journey. Information gained from these conferences was informative and led to new relationships and fresh ideas. One strategy developed as a result of the phone conferences was lunch-nlearn sessions, offered live once a month on Elluminate!, a real-time Web session. The lunch-n-learn topics are designed to answer specific requests by the NNPN participants and are recorded for later viewing for those unable to attend the live session. The majority of sessions are pre- pared and presented by nurses in member organizations who have a passion for, and expertise in, a particular topic area.

\section{EVALUATION}

The majority of work of the NNPN from 2005 through 2008 focused on completing the initial objectives of the four workgroups (e.g., development of the Web site, educational modules, and the Organizational Implementation Toolkit), developing relationships across NNPN hospitals (e.g., small group-phone conferences, live lunch-n-learn sessions, participation in workgroups), and providing research opportunities to meet the expressed needs of the nurses involved in the NNPN (e.g., RWJ and AONE research studies). While this work continues, the NNPN is moving to evaluate the effectiveness of the NNPN as a whole, and also various NNPN component activities.

Since the review of the Web site in spring 2008, substantial changes have been made to increase the usability of the Web site. This has resulted in a growth from March 2008 (400 registered users, an average of 1,000 visits to the site per month), when the initial study was conducted, to April 2009 (725 registered users, over 3,000 visits per month). New systems are in place to track which individual areas of the Web site attract the most use. Feedback from nurses in member hospitals suggests the Web site is being used in a variety of ways. Comments from various organizations include "we are changing the culture of nursing with our EBP and Research Council using the NNPN Web site as a source of education modules and resources for EBP. Completion of modules and quarterly review of the Web site is a requirement for our council members"; "The evidence-based practice programs and educational resources add richness to the work we are already doing and give us new ideas in how we can care for patients. When we find projects that are similar to what we are doing or trying to do, we don't always have to recreate the wheel"; "... nursing (at our organization) has been pleased to have the NNPN site as a forum for us to share our work, to receive information from other organizations who are working on similar issues, and hear their feedback."

In order to understand the effectiveness of the NNPN in improving patient outcomes, two research grants have been submitted. These grants propose to use both qualitative and quantitative methodology to increase understanding of mechanisms that foster dissemination and implementation of scientific findings and will evaluate the effectiveness of the NNPN in fostering adoption of EBPs for care of hospitalized adults. The studies will evaluate if use of the NNPN differs by organizational characteristics 
and also what components of the NNPN users find helpful in acute care settings to improve patient outcomes.

In addition, support from the VA CRIISP Center of Excellence is providing funding to begin several implementation projects across both VA and non-VA sites. These projects will include evaluation of the effectiveness of various NNPN activities in improving patient outcomes.

\section{FUTURE PLANS}

In order to maximize the effectiveness of the NNPN, new initiatives are under way. We are in the process of building closer interactive relationships and increasing the value to NNPN members through

- Monthly phone conferences with subgroups.

- Initiating projects identified by these same subgroups.

- Interactive Web-based training sessions on topics requested.

- Continued identification of research priority topics with funding.

- Increased number of face-to-face meetings and training sessions.

- Online venue for "publication" of gray literature.

- Building the infrastructure to handle the increase in demand.

- Identifying methods for evaluating the effectiveness of the NNPN network.

Although much has been accomplished, much work remains to increase the rate, extent, and sustainability of EBP both nationally and internationally. Organizational context variables impacting adoption need to be identified, along with identifying the effectiveness of various strategies used to promote EBP use. Because of the diverse nature of the member organizations, we envision many opportunities to increase current knowledge in these areas. Evaluating the effectiveness of the NNPN activities remains a high priority and ongoing process; evaluation data provide valuable information for our sites to maximize resource use, add to the body of knowledge of implementation science in general, and evaluate the feasibility of using multisite nursing collaboratives to improve patient care.

The NNPN continues to have requests from new organizations both nationally and internationally to join and is excited about the possibility of expanding the reach of the network to improve patient outcomes through use of evidence-based nursing practice as infrastructure support expands. Success of the NNPN depends on the continued active involvement and participation of members, both at an organizational and individual level. Member organi- zations provide a wealth of information, experience, and enthusiasm that will move the NNPN closer to the goal of improving patient outcomes through shared knowledge and learning.

\section{References}

Ayers L.R., Beyea S.C., Godfrey M.M., Harper D.C., Nelson E.C. \& Batalden P.B. (2005). Quality improvement learning collaboratives. Quality Management in Health Care, 14(4), 234-247.

Berwick D.M. (2003). Disseminating innovations in health care. Journal of the American Medical Association, 289(15), 1969-1975.

Cullen L., Greiner J., Greiner J., Bombei C. \& Comried L. (2005). Excellence in evidence-based practice: Organizational and unit exemplars. Critical Care Nursing Clinics of North America, 17(2), 127-142.

Cullen L. \& Titler M.G. (2004). Promoting evidence-based practice: An internship for staff nurses. Worldviews on Evidence-Based Nursing, 1(4), 215-223.

Fremont A.M., Joyce G., Anaya H.D., Bowman C.C., Halloran J.P., Chang S.W., Bozzette S.A. \& Asch S.M. (2006). An HIV collaborative in the VHA: Do advanced HIT and one-day sessions change the collaborative experience? Joint Commission Journal on Quality and Patient Safety/Joint Commission Resources, 32(6), 324-336.

Lesser E. \& Storck J. (2001). Communities of practice and organizational performance. IBM Systems Journal, 40(4), 831-941.

Mittman B.S. (2004). Creating the evidence base for quality improvement collaboratives. Annals of Internal Medicine, 140(11), 897-901.

Needleman J., Buerhaus P.I., Stewart M., Zelevinsky K. \& Mattke S. (2006). Nurse staffing in hospitals: Is there a business case for quality? Health Affairs, 25(1), 204211.

Stetler C.B., Legro M.W., Rycroft-Malone J., Bowman C., Curran G., Guihan M., Hagedorn H., Pineros S. \& Wallace C.M. (2006). Role of "external facilitation" in implementation of research findings: A qualitative evaluation of facilitation experiences in the veterans health administration. Implementation Science: IS, 1:23.

Titler M.G. (2002). Toolkit for promoting evidence-based practice. Iowa City, IA: University of Iowa Hospitals and Clinics.

Titler M.G. \& Everett L.Q. (2001). Translating research into practice. Considerations for critical care investigators. Critical Care Nursing Clinics of North America, 13(4), 587-604.

Wenger E. (1998). Communities of practice learning, meaning, and identity. New York: Cambridge University Press. 
Wenger E., McDermott R. \& Snyder W. (2002). Cultivating communities of practice. Cambridge, MA: Harvard Business School Press.

Wilson T., Berwick D.M. \& Cleary P.D. (2003). What do collaborative improvement projects do? Experience from seven countries. Joint Commis- sion Journal on Quality and Safety, 29(2), 8593.

Young P.C., Glade G.B., Stoddard G.J. \& Norlin C. (2006). Evaluation of a learning collaborative to improve the delivery of preventive services by pediatric practices. Pediatrics, 117(5), 1469-1476. 